\title{
Adenosine Kinase Deficiency: Report and Review
}

Henk J. Blom² Anna Wedell3,4,5,6

Majid Alfadhel ${ }^{1}$

\author{
Alhanouf Alhusani ${ }^{1} \quad$ Abdulrahman Obaid ${ }^{1}$ \\ ${ }^{1}$ Division of Genetics, Department of Pediatrics, King Abdullah \\ International Medical Research Centre, King Saud bin Abdulaziz \\ University for Health Sciences, King Abdulaziz Medical City, Riyadh, \\ Saudi Arabia \\ 2 Department of Internal Medicine, VU University Medical Center, \\ Amsterdam, the Netherlands \\ ${ }^{3}$ Division of Metabolic Diseases, Department of Laboratory Medicine, \\ Karolinska Institute, Stockholm, Sweden \\ ${ }^{4}$ Department of Molecular Medicine and Surgery, Science for Life \\ Laboratory, Karolinska Institute, Stockholm, Sweden \\ ${ }^{5}$ Centre for Inherited Metabolic Diseases, Karolinska University \\ Hospital, Stockholm, Sweden \\ ${ }^{6}$ Max Planck Institute Biology of Ageing, Karolinska Institute \\ Laboratory, Stockholm, Sweden
}

Neuropediatrics 2019;50:46-50.
Address for correspondence Majid Alfadhel, MD, MHSC, FCCMG, Division of Genetics, Department of Pediatrics, King Saud bin Abdulaziz University for Health Sciences, King Abdulaziz Medical City, PO Box 22490, Riyadh 11426, Saudi Arabia (e-mail: dralfadhelm@gmail.com).

\begin{abstract}
Keywords

- methionine

- ADK

- adenosine kinase deficiency

- adenosine

- inborn errors of methionine

Adenosine kinase (ADK) deficiency (OMIM [online mendelian inheritance in man]: 614300 ) is an autosomal recessive disorder of adenosine and methionine metabolism, with a unique clinical phenotype, mainly involving the central nervous system and dysmorphic features. Patients usually present early in life with sepsis-like symptoms, respiratory difficulties, and neonatal jaundice. Subsequently, patients demonstrate hypotonia and global developmental delay. Biochemically, methionine is elevated with normal homocysteine levels and the diagnosis is confirmed through molecular analysis of the $A D K$ gene. There is no curative treatment; however, a methionine-restricted diet has been tried with variable outcomes. Herein, we report a 4-year-old Saudi female with global developmental delay, hypotonia, and dysmorphic features. Interestingly, she has a tall stature, developmental dysplasia of the hip, optic nerve gliosis, and tigroid fundus. We found a mutation not reported previously and we compared the current case with previously reported cases. We alert clinicians to consider ADK deficiency in any neonate presenting with global developmental delay, hypotonia, dysmorphic features, and high methionine levels.
\end{abstract}

\section{Introduction}

Adenosine kinase (ADK) deficiency (OMIM [online mendelian inheritance in man]: 614300) is an autosomal recessive inborn error of methionine and adenosine metabolism. It was first described by Bjursell et al in 2011 who reported two Swedish children with global developmental delay, hypermethioninemia, and liver impairment. ${ }^{1}$ It is caused by a homozygous or compound heterozygous mutation in the $A D K$ gene which encodes for the ADK enzyme. ${ }^{2}$ The ADK enzyme is essential for the phosphorylation of adenosine to adenosine monophosphate. ${ }^{3}$ In addition, it removes adenosine which is critical for S-adenosylhomocysteine (AdoHcy) hydrolase in methylation reactions, as it maintains S-adenosylmethionine (AdoMet) in trans methylation-dependent paths, where adenosine has to be continually removed. ${ }^{4}$ Under physiological status, adenosine is produced through the transmethylation of methionine, via AdoMet and AdoHcy. Subsequently, AdoHcy hydrolysis is achieved by SAdoHcy hydrolase to generate Hcy and adenosine. ${ }^{5}$ Hcy is remethylated again to methionine or irreversibly transsulfurated to cystine by cystathionine $\beta$-synthase, while received

July 1,2018

accepted after revision

October 15, 2018

published online

November 26, 2018 (c) 2019 Georg Thieme Verlag KG Stuttgart · New York
DOI https://doi.org/ 10.1055/s-0038-1676053. ISSN 0174-304X. 
the adenosine is removed by deamination into inosine via adenosine deaminase (prenatally) and into adenosine monophosphate via phosphorylation (postnatally) by ADK., ${ }^{6,7}$ Accumulation of adenosine will cause elevated S-AdoHcy which is a strong inhibitor of most methylation reactions. Consequently, levels of S-AdoMet and methionine can become increased.

The clinical features of ADK deficiency include global psychomotor retardation/delay, muscular hypotonia, dysmorphic features (especially frontal bossing), mild to severe hepatic dysfunction, and epilepsy. ${ }^{1,2}$ Laboratory findings include high levels of liver enzymes, hyperbilirubinemia, hypermethioninemia, and elevated plasma AdoHcy and AdoMet. Neuroradiological abnormalities include brain atrophy, hydrocephalus, nonspecific white matter changes, and delayed myelination. Diagnosis requires the aforementioned clinical and biochemical abnormalities and confirmation through molecular analysis of the $A D K$ gene. ${ }^{1,2,5}$ There is no curative treatment. However, some previously reported cases were treated through a methionine-restricted diet and in addition to support the treatment, it included periodic monitoring of neurological and hepatic function, physiotherapy, occupational therapies, and long-term rehabilitation. These treatments resulted in variable outcomes. ${ }^{2,5}$ ADK deficiency has been reported in different countries including Sweden, Malaysia, Germany, and Iran. In the current report, we describe the first Saudi case of ADK deficiency, with optic nerve gliosis, tigroid fundus, and a tall stature that has not yet been associated with ADK deficiency. A novel frameshift duplication was found. We compare the presented case with the previously reported cases.

\section{Case Report}

The case concerns a 4-year-old female who is the fifth child of parents who are first cousins. She was born at 35 weeks of gestation through caesarean section because of preterm labor and a previous caesarean section with an Apgar's score 7 and 8 at 1 and 5 minutes, respectively. Her birth weight was $2.9 \mathrm{~kg}$ (75-90th percentile), length: $49 \mathrm{~cm}$ (7590th percentile), and head circumference (HC): $33.5 \mathrm{~cm}$ (75-90th percentile). A few hours after birth, she developed tachypnoea, respiratory distress, and hypoactivity, and therefore was transferred to an Intermediate Care Nursery and was connected to a nasal cannula and treated using antibiotics and other supportive measures. On examination, she was found to be hypotonic with poor sucking and she developed jaundice. Therefore, the neurology department became involved and magnetic resonance imaging (MRI) of the brain showed delayed myelination and a left subependymal cyst. At this time, a metabolic genetics service became involved and the review for initial newborn screening was unremarkable. However, plasma amino acid levels at 18 days of life showed a high methionine level of 123 $\mu \mathrm{mol} / \mathrm{L}$ (normal range $11-27 \mu \mathrm{mol} / \mathrm{L}$ ) with a normal homocysteine level. The patient improved, and demonstrated spontaneous breathing, good sucking, weight gain, and tolerated feeding, and therefore was discharged after 1 month, with follow-up occurring in a clinic. At the age of 12 months, the patient was readmitted with poor feeding, decreased activity, fever $\left(38.9^{\circ} \mathrm{C}\right)$, symptoms of an upper respiratory tract infection, and vomiting. Developmentally, she was bed-bound, with no head support. She was unable to sit or crawl (functioning as 2 months). Her weight was $9.5 \mathrm{~kg}$ (25th percentile), length $79 \mathrm{~cm}$ (95th percentile), and $\mathrm{HC}$ was $49 \mathrm{~cm}$ (>95th percentile). She demonstrated subtle dysmorphic features (frontal bossing, high forehead, deep-seated eyes, and depressed nasal bridge; (-Fig. 1). A neurological examination showed generalized hypotonia. The investigation also revealed persistent hypermethioninemia (methionine levels at approximately $1,500 \mu \mathrm{mol} / \mathrm{L}$ ). The diagnosis of ADK deficiency was confirmed through whole-exome sequencing, (WES) which revealed a novel homozygous duplication in
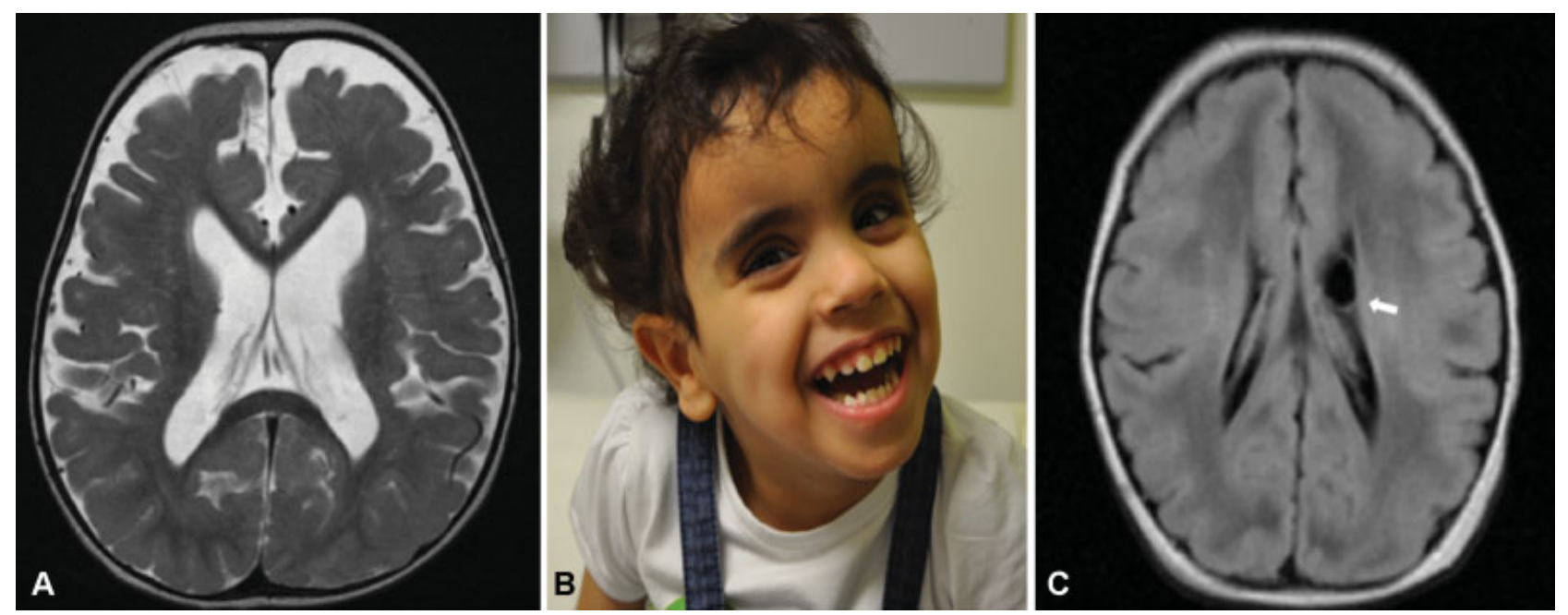

Fig. 1 Dysmorphic features and brain MRI features of ADK deficiency. (A) An axial T2-weighted image shows delayed myelination. (B) Frontal bossing, high forehead, deep-seated eyes, and depressed nasal bridge. (C) An axial FLAIR image shows a small subependymal cyst at the left lateral ventricle. ADK, adenosine kinase; MRI, magnetic resonance imaging; FLAIR, fluid attenuated inversion recovery. 
exon 9 of the ADK gene; NM_006721.3 [c.813dup (p. Asn272Glufs*16)]. This variant was detected in the heterozygous state in both parents and was not detected in a healthy brother of the patient. The patient stabilized following supportive measures and was discharged. An MRI scan of the brain, repeated at 22 months of age, showed cerebral atrophy, delayed myelination, and a left subependymal cyst ( - Fig. 1). Subsequently, the patient continued to demonstrate global developmental delay, dysmorphic features, no history of seizure, and was admitted to hospital several times because of recurrent chest infections. Additionally, the patient demonstrated a mild elevation in transaminases: aspartate aminotransferase levels (AST) ranged between 47 and $200 \mathrm{U} / \mathrm{L}$ (normahealthy: 5-34 U/L) while alanine aminotransferase (ALT) ranged between 77 and $194 \mathrm{U} / \mathrm{L}$ (normal 5-55 U/L). However, there was no hepatomegaly and the synthetic function of the liver was normal.

Currently, at 48 months of age, she is wheelchair-bound with severe hypotonia and global developmental delay, she is unable to sit unsupported and reach or transfer objects, babbles, she is friendly with family and strangers, still wears diapers, and demonstrates good vision and hearing (functioning at 5-6 months of age). Her current weight is $13.7 \mathrm{~kg}$ (25-50th percentile), length $112 \mathrm{~cm}$ (>95th percentile), HC: $52.5 \mathrm{~cm}$ (>95th percentile), and she is macrocephalic with the same subtle dysmorphic features. She has a squint and an ophthalmological evaluation revealed optic nerve gliosis and tigroid fundus with normal pupil size. A neurological examination showed head lag, generalized hypotonia, and normal reflexes. A respiratory, cardiovascular, and gastrointestinal examination were unremarkable and the patient has normal female external genitalia. A skeletal survey showed bilateral dysplasia of the hip with subluxation without shorting or contractures. An auditory brainstem response assessment, echocardiogram, and abdominal ultrasound were normal. Of note, all the following biochemical and genetic investigations were unremarkable: acylcarnitine profile, ammonia, lactic acid, creatine kinase levels, total homocysteine, urine amino acids, urine organic acids, liver enzymes, coagulation profile, lipid profile, chromosomal analysis, comparative genomic hybridization (CGH) microarray, and DNA (deoxyribonucleic acid) molecular testing for the MAT1A gene. Unfortunately, we were unable to measure AdoHcy levels or AdoMet levels because of difficulty in finding a laboratory performing such tests and poor parental compliance. In addition, a methioninerestricted formula was refused by the parents. Interestingly, methionine levels over the past 2 years became lower spontaneously without any intervention (59-68, 68-77 $\mu \mathrm{mol} / \mathrm{L}$ respectively).

\section{Discussion}

Hypermethioninemia is a biochemical marker of inherited inborn errors of metabolism in the methylation pathway, such as methionine adenosyltransferase I/III (MAT I/III) deficiency, S-AdoHcy hydrolase (SAHH) deficiency, and cystathionine $\beta$-synthase (CBS) deficiency. And secondary due to, fumarylacetoacetate hydrolase (FAH) deficiency, citrin deficiency, and mitochondrial depletion syndrome caused by mutations in the MPV17 and DGUOK genes., The most common cause of hypermethioninemia is liver disease, next to low birth weight and prematurity. ${ }^{9}$

- Table 1 summarizes the clinical characteristic of previously reported patients with ADK deficiency, including the current case. To date, 19 cases have been reported in the literature. ADK deficiency is pan-ethnic. Patients from Sweden, Malaysia, Turkey, Kuwait, Italy, Morocco, Germany, and Iran have been reported. The current report details the first Saudi patient to date. A total of $90 \%$ of cases present in the neonatal period, indicating an early onset with sepsis-like symptoms, hypoglycemia, and neonatal jaundice in $84 \%$ of patients. All patients demonstrate global developmental delay, frontal bossing, and hypotonia. The most common phenotype includes dysmorphic features in the form of frontal bossing, deep-seated eyes, hypertelorism, a high forehead, a depressed nasal bridge, thin sparse hair, and slender hands and feet. Macrocephaly is found in at least half of patients. Seizure presents in approximately $68 \%$ of cases, and the type is nonspecific consisting of both partial and generalized epilepsy with a variable age of onset. Hepatological signs consist of elevated transaminases (90\%) and hepatic fibrosis (32\%), while hepatomegaly was found only in one patient. Cardiac defects, including pulmonary stenosis, atrial septal defects, coarctation of the aorta, ventricular septal defects, and patent ductus arteriosus, have been reported. Interestingly, the current case showed no hepatomegaly, hepatic fibrosis, or seizures. Approximately $60 \%$ of patients suffer from failure to thrive and short stature but the presented case demonstrates tall stature. Other features present in a few cases include strabismus, undescended testis, retinal dystrophy, abnormal dentition, cholelithiasis, neurogenic bladder, and megaloblastic anemia. The present patient differs from the previously reported cohort through the findings of developmental dysplasia of the hip, optic nerve gliosis, and tigroid fundus. These findings expand the phenotype of ADK deficiency.

The $A D K$ gene has been mapped to chromosome $10 \mathrm{q} 22.2$, spans approximately $550 \mathrm{~kb}$ of genomic DNA, and consists of 11 exons. The mutation spectrum of ADK deficiency is extremely heterogeneous. The most common type is missense mutation (50\%). Other variations include deletion, nonsense, and deletion-insertion mutations. This is the first report of a duplication creating a frameshift starting at codon Asn272 and ending in a stop codon 15bp downstream. This variant is not described in the Exome Aggregation Consortium, Exome Sequencing Project, or 1,000 genomes browser, as well as a database of 2000 in-house ethnically matched xomes supports its pathogenicity. In ADK deficiency, there appears to be no clear genotype-phenotype correlation.

Since methionine is elevated in this disorder, substrate reduction therapy through a methionine-restricted diet (MRD) may be reasonable and $42 \%$ of the cohort was treated through an MRD with variable efficacy. In several patients, an 
Table 1 Summary of the clinical characteristics of previously reported patients with ADK deficiency compared with the current case

\begin{tabular}{|c|c|c|c|c|c|}
\hline Authors & $\begin{array}{l}\text { Bjursell et al, } \\
2011\end{array}$ & $\begin{array}{l}\text { Staufner et al, } \\
2016\end{array}$ & $\begin{array}{l}\text { Shakiba et al, } \\
2016\end{array}$ & $\begin{array}{l}\text { Current study, } \\
2018\end{array}$ & Total (\%) \\
\hline Number of cases & 6 & 11 & 1 & 1 & 19 \\
\hline Male:female & $4: 2$ & $4: 7$ & $0: 1$ & $0: 1$ & $8: 11$ \\
\hline Ethnicity & $\begin{array}{l}\text { Two Swedish and } \\
\text { four Malaysian }\end{array}$ & $\begin{array}{l}\text { Four Turkish, } \\
\text { two Kuwaiti, one } \\
\text { Italian, one } \\
\text { Moroccan, one } \\
\text { German, two } \\
\text { Iranian }\end{array}$ & Iranian & Saudi & \\
\hline Age at diagnosis (y & $8-24$ & $1.9-29$ & 3 & 1 & $1-24$ \\
\hline Neonatal onset & $6 / 6$ & $9 / 11$ & + & + & $17 / 19(90)$ \\
\hline $\begin{array}{l}\text { Developmental } \\
\text { delay }\end{array}$ & $6 / 6$ & $11 / 11$ & + & + & $19 / 19(100)$ \\
\hline Frontal bossing & $6 / 6$ & $11 / 11$ & + & + & $19 / 19(100)$ \\
\hline Hypotonia & $6 / 6$ & $11 / 11$ & + & + & $19 / 19(100)$ \\
\hline Seizure & $6 / 6$ & $7 / 11$ & - & - & $13 / 19(68)$ \\
\hline Neonatal jaundice & $5 / 6$ & $9 / 11$ & + & + & $16 / 19(84)$ \\
\hline $\begin{array}{l}\text { Elevated } \\
\text { transaminases }\end{array}$ & $6 / 6$ & $9 / 11$ & + & + & $17 / 19(90)$ \\
\hline Hepatic fibrosis & $1 / 6$ & $4 / 11$ & + & - & $6 / 19(32)$ \\
\hline Hepatomegaly & - & - & + & - & $1 / 19(5)$ \\
\hline Hypertelorism & $6 / 6$ & $7 / 11$ & + & + & $17 / 19(90)$ \\
\hline Sparse, thin hair & - & $4 / 11$ & + & - & $5 / 19(26)$ \\
\hline $\begin{array}{l}\text { Short stature and } \\
\text { FTT }\end{array}$ & $6 / 6$ & $5 / 11$ & - & - & $11 / 19(58)$ \\
\hline Cardiac defect & $3 / 6$ & $5 / 11$ & + & - & $9 / 19(47)$ \\
\hline $\begin{array}{l}\text { Sensorineural } \\
\text { hearing loss }\end{array}$ & $2 / 6$ & - & - & - & $2 / 19(10)$ \\
\hline $\begin{array}{l}\text { Methionine } \\
\text { restricted diet }\end{array}$ & - & $7 / 11$ & + & - & $8 / 19(42)$ \\
\hline Prognosis & $\begin{array}{l}\text { One patient died } \\
\text { at } 10 \text { y after a } \\
\text { refractory seizure. } \\
\text { The others sur- } \\
\text { vived until adult- } \\
\text { hood with global } \\
\text { developmental } \\
\text { delay }\end{array}$ & $\begin{array}{l}\text { One patient died } \\
\text { at } 15 \text { mo. The } \\
\text { others survived } \\
\text { and some reached } \\
\text { adulthood with } \\
\text { global develop- } \\
\text { mental delay }\end{array}$ & $\begin{array}{l}\text { Global develop- } \\
\text { mental delay }\end{array}$ & $\begin{array}{l}\text { Global develop- } \\
\text { mental delay }\end{array}$ & \\
\hline Other features & $\begin{array}{l}\text { Macrocephaly in } \\
5 / 6 . \text { Slender hands } \\
\text { and feet }\end{array}$ & $\begin{array}{l}\text { Undescended tes- } \\
\text { tis, strabismus, ret- } \\
\text { inal dystrophy, } \\
\text { abnormal denti- } \\
\text { tion, cholelithiasis, } \\
\text { MA, slender hands } \\
\text { and feet }\end{array}$ & $\begin{array}{l}\text { Recurrent infec- } \\
\text { tion, neurogenic } \\
\text { bladder, megalo- } \\
\text { blastic anemia }\end{array}$ & $\begin{array}{l}\text { Macrocephaly, tall } \\
\text { stature, optic nerve } \\
\text { glioma, tigroid } \\
\text { fundus, develop- } \\
\text { mental dysplasia of } \\
\text { the hips, lax joint, } \\
\text { slender hands and } \\
\text { feet }\end{array}$ & \\
\hline
\end{tabular}

Abbreviation: ADK, adenosine kinase; FTT, failure to thrive; MA, megaloblastic anemia.

improvement in cognitive function, developmental delays, and speech were reported while in others treatment was ineffective. The current patient was not treated through an MRD. However, methionine levels decreased spontaneously over the past 2 years. The early onset of ADK deficiency in the first few days of life indicates that the pathology may start in utero and this may explain why MRD has been largely ineffective in this disorder. 
Two patients died in the reported cohort, while the others survived into the third decade, demonstrating that this disorder is generally not lethal and patients can live but with global developmental delay.

\section{Conclusion}

We expand the phenotype of ADK deficiency to include tall stature, developmental dysplasia of the hip, optic nerve gliosis, and tigroid fundus. We have reported a novel mutation, and we compared the current case with previously reported cases. ADK deficiency consists of a triad of global developmental delay, hypotonia, and frontal bossing. Macrocephaly and hypertelorism are common findings; and this disease has a variable degree of hepatic and cardiac features, and early onset of the disease is characteristic. Moreover, international registries such as E-HOD (European Network and Registry for Homocystinurias and Methylation Defects; http://www.ehod.org/), which document more cases of the ADK gene defect, will lead to a better understanding of the natural history of this inborn error of metabolism and facilitate well-designed, largesample clinical trials which may aid in improving the management of this genetic disorder.

\section{Author Contributions}

A.A.: wrote the first draft and edited the subsequent version of the manuscript.

A.O.: collected the clinical data and edited the manuscript. A.W.: edited the manuscript and reviewed the clinical data.

H.J.B.: edited the manuscript and reviewed the clinical data.

M.A.: supervised the work associated with preparing, writing, and submitting the manuscript and contributed to the clinical diagnosis and management of the patient.

All authors have read and approve the final draft of the manuscript.

Conflict of Interests

None.

\section{Funding Sources}

No funding for this article from any institution or agency.

Ethics Approval and Consent to Participate

This study was approved by the institutional review board office at King Abdullah International Medical Research Centre (KIMARC; study number: RC16/113/R).

\section{Consent for Publication}

Written consent was obtained from the parents.

\section{Acknowledgments}

We are grateful to the patient and her family for their genuine support.

\section{References}

1 Bjursell MK, Blom HJ, Cayuela JA, et al. Adenosine kinase deficiency disrupts the methionine cycle and causes hypermethioninemia, encephalopathy, and abnormal liver function. Am J Hum Genet 2011;89(04):507-515

2 Staufner C, Lindner M, Dionisi-Vici C, et al. Adenosine kinase deficiency: expanding the clinical spectrum and evaluating therapeutic options. J Inherit Metab Dis 2016;39(02):273-283

3 Spychala J, Datta NS, Takabayashi K, et al. Cloning of human adenosine kinase cDNA: sequence similarity to microbial ribokinases and fructokinases. Proc Natl Acad Sci U S A 1996;93(03): 1232-1237

4 Boison D. Adenosine kinase: exploitation for therapeutic gain. Pharmacol Rev 2013;65(03):906-943

5 Barić I. Inherited disorders in the conversion of methionine to homocysteine. J Inherit Metab Dis 2009;32(04):459-471

6 Baric I, Fumic K, Glenn B, et al. S-adenosylhomocysteine hydrolase deficiency in a human: a genetic disorder of methionine metabolism. Proc Natl Acad Sci U S A 2004;101(12):4234-4239

7 Boison D, Scheurer L, Zumsteg V, et al. Neonatal hepatic steatosis by disruption of the adenosine kinase gene. Proc Natl Acad Sci U S A 2002;99(10):6985-6990

8 Shakiba M, Mahjoub F, Fazilaty H, et al. Adenosine kinase deficiency with neurodevelopemental delay and recurrent hepatic dysfunction: A case report. Adv Rare Dis 2016;3:3

9 Mudd SH. Hypermethioninemias of genetic and non-genetic origin: A review. Am J Med Genet C Semin Med Genet 2011; $157 \mathrm{C}(01): 3-32$ 